To be published in Applied Optics:

Title: $\quad$ Comparative study of ring and random cavities for fiber lasers

Authors: Montserrat Fernandez-Vallejo, Sergio Rota-Rodrigo, and Manuel Lopez-Amo Accepted: 25 April 2014

Posted: 28 April 2014

Doc. ID: 205182

(C) 2014 Optical Society of America. One print or electronic copy may be made for personal use only. Systematic reproduction and distribution, duplication of any material in this paper for a fee or for commercial purposes, or modifications of the content of this paper are prohibited. 


\title{
Comparative study of ring and random cavities for fiber lasers
}

\author{
Montserrat Fernandez-Vallejo ${ }^{1 *}$, Sergio Rota-Rodrigo ${ }^{1}$ and Manuel Lopez-Amo ${ }^{1}$ \\ ${ }^{1}$ Department of Electric and Electronic Engineering, Universidad Publica de Navarra, 31006 Pamplona, Spain \\ ${ }^{*}$ Corresponding author: Montserrat.fernandez@unavarra.es
}

Received Month X, XXXX; revised Month X, XXXX; accepted Month X,

XXXX; posted Month X, XXXX (Doc. ID XXXXX); published Month X, XXXX

\begin{abstract}
An experimental comparison of three fiber laser structures with the same Raman gain medium is presented in order to establish the main pros and cons of each basic scheme. The first fiber laser is based on a hybrid ringrandom fiber laser; the second one is a purely ring fiber laser and the last one is a random fiber laser. Several aspects have been taken into account in the study. Firstly, from the optical point of view, the parameters of interest compared are output power, lasing threshold, slope efficiency, power fluctuations and finally, the longitudinal modes have been analyzed. And secondly, the possible utilization of fiber lasers in digital modulated
\end{abstract} optical communication systems is also studied.

OCIS codes: (060.3510) Fiber lasers; (140.3550) Raman lasers; (140.3560) Ring lasers.

http://dx.doi/org/10.1364/AO.99.099999

\section{Introduction}

Raman fiber lasers (RFLs) use stimulated Raman scattering (SRS) in optical fiber to shift the wavelength of the input pump laser to another desired wavelength. In spite of the fact that the first RFLs were demonstrated in the 1970s, they were inefficient and low output power devices till the advent of fiber Bragg gratings (FBGs) which transforms Raman fiber lasers in a challenging technology. From that moment on, experimental and theoretical results have been presented demonstrating the possibilities of this technology in some applications such as in the telecommunication field or fiber optic sensors. [1-6]

Due to the unique characteristic of Raman amplification which can provide broad gain bands in any wavelength region by selecting the pump wavelengths, the inhomogeneous broadened gain of stimulated Raman scattering and the availability of highpower laser diode pumps, fiber Raman lasers become of great importance in different applications: wavelength division multiplexing (WDM), instrument testing, optical fiber sensors, sensor network multiplexing schemes, ultra-long and remote sensor networks, optical sensing processing and spectroscopy. [3, 7-10]

As any fiber laser, Raman fiber lasers basically consist of a gain medium that provides amplification and an optical cavity that traps the light and therefore a positive feedback is created. Different devices have been used with this purpose generating multiwavelength outputs: from Fabry-Perot filters, or highbirefringence fibers, to the traditional fiber Bragg gratings [10, 11]. However, since few years ago, a new kind of Raman fiber lasers appeared: random fiber lasers which are mainly characterized by mirrorless cavities, in other words, the previously mentioned devices are not necessary to trap the light.
This fact entails the first advantage of this kind of fiber lasers, the required technology is relatively simple and they have low production costs. [12-22]

The principle of operation of random fiber lasers is based on multiple scattering events which are amplified through Raman effect. The laser condition in random fiber lasers is fulfilled thanks to the accumulation of multiple Rayleigh scattering along with its amplification over a long distance, in spite of the fact that this process itself is extremely weak in the fiber core. Thus, optical scattering, considered as an adverse process, has turned into a useful effect. [12-22]

Up to now, some published fiber Raman lasers works based on stimulated Raman scattering had certain component random which was ignored. The effect was known by the research community but, maybe, it would not have been given the importance that deserves [9, 23-26]= Nowadays, it has been demonstrated that the accumulation of small, but amplified Rayleigh scattering can effect directly to the behavior of Raman fiber lasers.

In this work, an experimental comparison of the behavior of three basic fiber lasers is presented. The first Raman fiber laser is based on a hybrid ring-random fiber laser; the second one is a purely ring fiber laser and the last one is a random fiber laser. The study is focused on, on the one hand, optical parameters such as output power, lasing threshold, slope efficiency, power fluctuations and finally, analysis of the modes. And, on the other hand, the possible applications of the proposed fiber lasers in the optical communication field are also analyzed through the modulation of the signal. 


\section{Experimental set-up}

Three fiber laser topologies are compared: the first one is based on a hybrid ring-random fiber laser; the second one is a ring fiber laser and the last one is a random fiber laser. Since the major goal of the paper is the comparison of the behavior of the three basic fiber laser configurations, the proposed schemes are as simple as possible. The three fiber lasers are depicted in Fig. 1 and their characteristics are described below:

The following aspects are common to all topologies:

- A section of $2.4 \mathrm{~km}$ dispersion compensating fiber (DCF) is used to achieve greater Raman gain per unit pump power. The Raman pump laser radiated at $1445 \mathrm{~nm}$ and could deliver up to 3 W. The pump power is launched to the system through a wavelength division multiplexer (WDM).

- The wavelength selection is carried out by means of a FBGs centered at $1545 \mathrm{~nm}$ with $0.2 \mathrm{~nm}$ bandwidth and $95 \%$ reflectivity.

- All the free terminations of all systems were immersed in refractive-index-matching gel to avoid undesired reflections. This issue can be particularly critical in the random fiber laser.

The initial configuration, the ring-random fiber laser, shown in Fig 1.a, has been chosen in such a way the behavior of the hybrid scheme could be studied. Blue arrows point out the optical path followed by the random fiber laser contribution, while green arrow indicates the direction of the fiber ring laser. But, afterwards, it is also possible to study individually the contributions of each basic fiber laser that compose the hybrid fiber laser. The pure-ring laser structure (Fig. 1.b) is obtained by adding an optical isolator to the previous structure and finally, the random fiber laser (Fig. 1.c) consists of the cavity section of the first structure wherein the random mirror was affecting the behavior of the hybrid configuration. The output of each laser (after the reflection in the FBG) was selected to reduce the effect of the noise in the optical signal to noise ratio (OSNR).

\section{Hybrid ring-random fiber laser}

The first fiber laser is based on a hybrid ring-random fiber laser (Fig. 1. (a)). On the one hand, the ring structure uses the backward spontaneous Raman scattering in order to generate a counterclockwise fiber laser whose wavelength operation is determined by the FBG. The random fiber laser, on the other hand, creates a linear cavity between the FBG and the DCF spool which works as a distributed mirror, thus, this laser uses the amplified Rayleigh scattering feedback through Raman effect. In conclusion, the output signal is composed of the contribution of two fiber lasers with different nature but operating at the same wavelength.

To extract a $5 \%$ of the laser output power from the ring, a $95 \%$ coupler is used and an optical spectrum analyzer is utilized to measure different parameters.

An optical isolator is also included between the optical couplers to ensure unidirectional operation of the ring laser avoiding spatial hole-burning (SHB) effect. but This position of the isolator does not obstruct the random fiber laser behavior.

The scheme of the random fiber laser also includes a $95 \%$ coupler which, at first sight, could seem is in excess. However, it has been added to make the comparison study under equal conditions of losses in each of the three fiber laser structures.

\section{Ring fiber laser}

The second laser has a purely ring topology. As it can be seen in Fig. 1. (a) and (b), both schemes are equal apart from an extra isolator located between the WDM and the $50 \%$ coupler. This isolator avoids the generation of the random fiber laser because the power only can travel in the counterclockwise, thus, it is not possible to have feedback from the random mirror.

\section{Random fiber laser}

Fig. 1. (c) depicts the schematic setup of the random fiber laser. This laser is based on a linear cavity formed of two different mirrors: the first one, the FBG which selects the emission wavelength and the second one, a distributed mirror created by the dispersion compensating fiber reel as it has been previously explained in sub-section A. It seems important to highlight that angled cleaves were used at the fiber end facets to eliminate reflections and ensure that the feedback is due only to the randomly distributed scattering [16]-

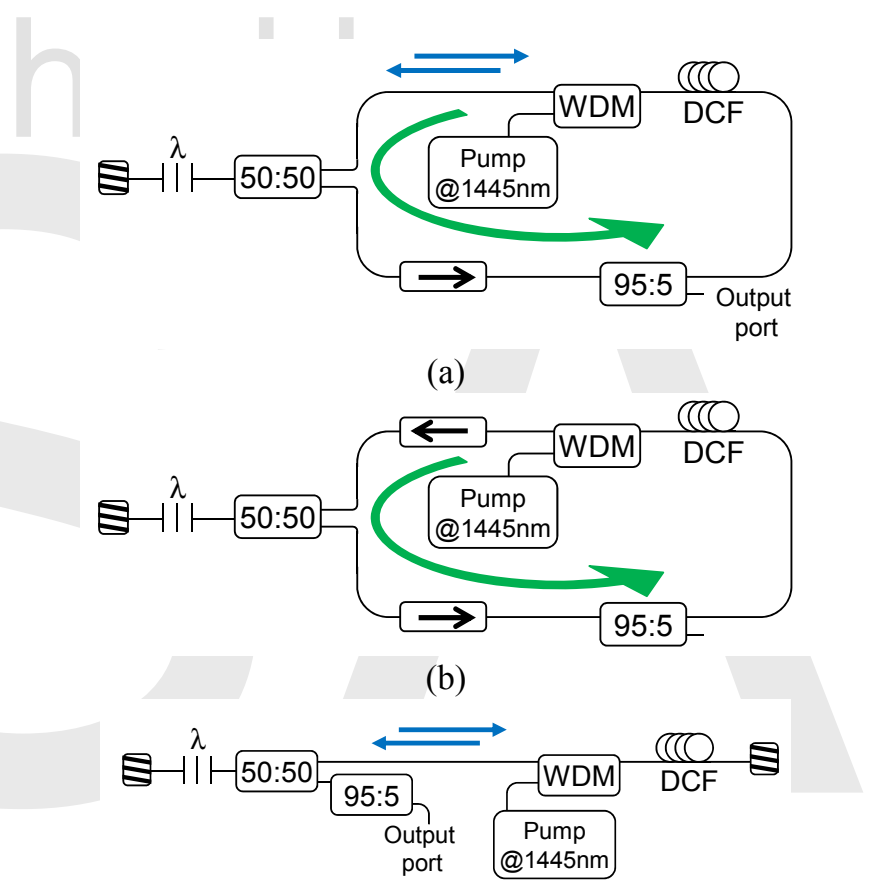

(c)

Fig. 1. Experimental set-up of: (a) the hybrid ring-random fiber laser; (b) the ring fiber laser; and (c) random fiber laser. DCF: Dispersion compensating fiber. WDM: wavelength-division multiplexer. Black arrow symbol: optical isolator. Blue arrows: Random laser feedback. Green arrows: Ring laser contributions

\section{Results}

The main goal of this work is the comparison of the three basic configurations taking into account different aspects. Firstly, from the optical point of view, the parameters of interest are: output power, lasing threshold, slope efficiency, power fluctuations and finally, analysis of the modes. And secondly, the applications of the fiber lasers in the optical communication field are also studied.

First of all, the output power evolution versus the Raman pump power launched to the system was measured using a power meter. The power progressions are shown in Fig. 2 and 
Fig. 3. Both figures show the same result in different scales in order to appreciate different aspects of the lasers outputs. One interesting parameter to analyze, from the results displayed on Fig. 2, is the pump power necessary to achieve the laser condition: at the beginning the output power increases with a low slope when pump power is also increased, but, afterwards there is a great leap with a small increment of pump power, thus the laser behavior is demonstrated. It is also observed from Fig. 2 and Fig. 3 that the threshold power of the hybrid ring-random laser is the lowest, around $390 \mathrm{~mW}$; the purely ring laser threshold is around $500 \mathrm{~mW}$ and finally, the all random mirror is the configuration which needs higher pump power, $650 \mathrm{~mW}$, to reach the lasing operation.

Regarding the output power and slope efficiency, the ring scheme offers the better characteristics followed by the hybrid laser and the random fiber laser is the worst option taking into account this parameters.

In conclusion, the combination of the ring and the random cavity improves the laser threshold due to a more efficiently use of the amplification, up to this point. However, when the laser condition is achieved, a lower final output power, in comparison with the ring laser, is obtained because some of the Raman amplification is used to amplify the signal that travels in clockwise direction that is finally lost at the isolator.

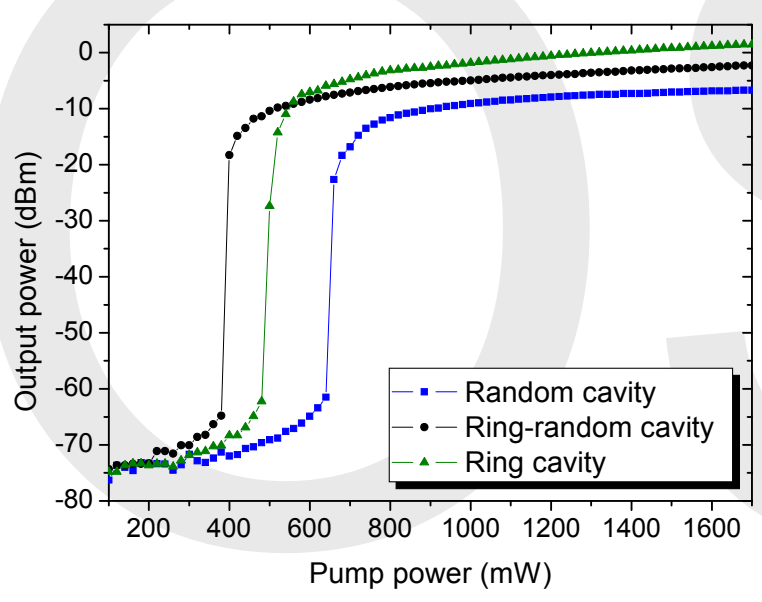

Fig. 2. Experimental demonstration of laser condition through the evolution of the output power versus pump power.

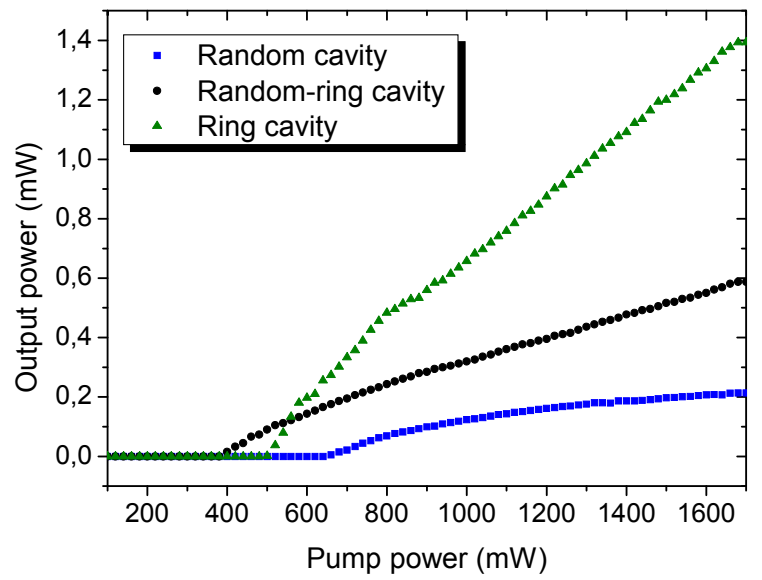

Fig. 3. Evolution of the output power versus pump power presenting the output power with a linear scale where the differences of threshold power and efficiency among the proposed fiber lasers are evident.
Fiber lasers are known to be susceptible to output power instabilities [9], particularly in multiwavelength fiber lasers but also, although to a lesser extent, in the case of only one emission line. This issue is especially critical because these instabilities can degrade the performance characteristics of a telecommunication or sensor multiplexing network based on a laser interrogation scheme. For this reason, the survey of the output power fluctuations of the proposed lasers is required, Fig. 4 shows the results. The instability is defined as the output power variation for a given interval of time and a specific confidence interval (CI), given as a percentage. The confidence interval (CI) is the estimated range of values where the parameter of interest is included [9]. It should be stressed that due to the non multiwavelength operation of the studied fiber lasers, the power fluctuations are lower than $0.1 \mathrm{~dB}$ in all the cases. The ring cavity offers the better stability, and the worst case comes from the hybrid ring-random fiber laser, this fact may be due to the gain competition established among signals amplified from the random and ring cavities.

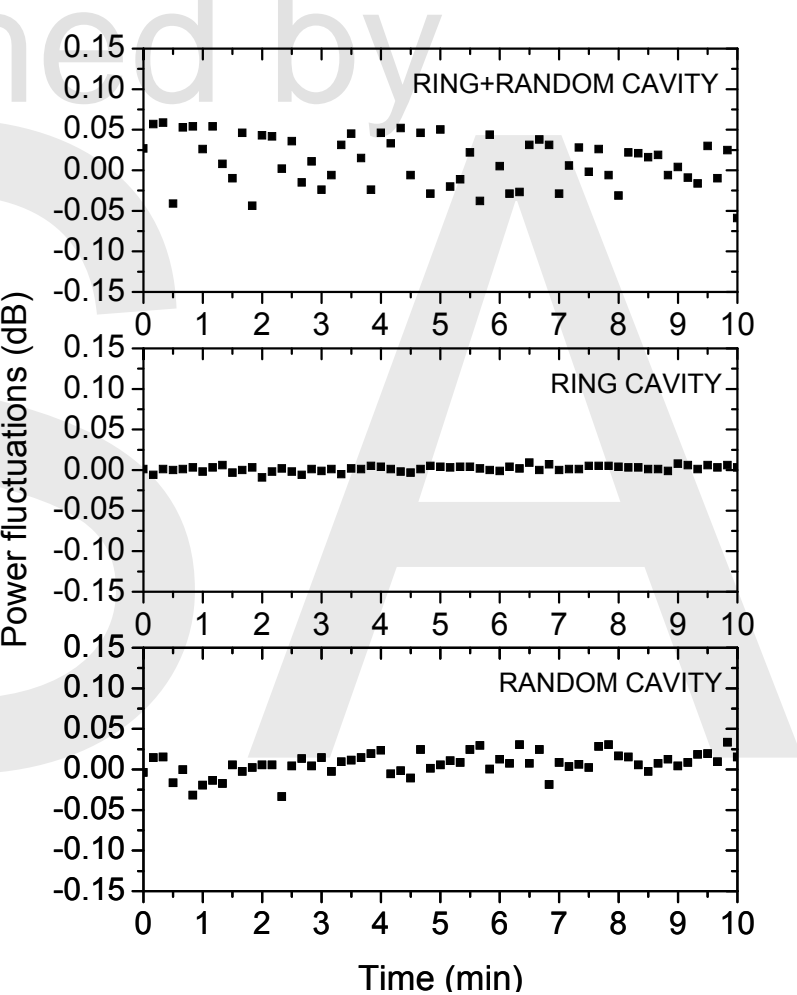

Fig. 4. Output power fluctuations of the three laser configurations versus time.

The behavior of the longitudinal modes in the cavity can be interesting in order to have a more complete understanding of the basic proposed schemes both in the extreme cases when the lasers are completely ring or random and in the hybrid ringrandom laser.

The purely ring laser generates longitudinal modes which are evenly spaced according to the following equation:

$$
\Delta f=\frac{c}{n L}
$$

where $\mathrm{c}$ is the speed of the light in vacuum, $\mathrm{n}$ is the refractive index of the fiber and $\mathrm{L}$ is the fiber length of the cavity. Thus, in the fiber ring laser the resonance longitudinal modes are spaced 
around $86.8 \mathrm{KHz}$. This distance is too low to be observed in our optical spectrum analyzers, thus the longitudinal modes were detected in the electrical domain using an electrical spectrum analyzer (ESA). As many modes are generated in the cavity due to the long cavity length, the ESA shows the beat among the longitudinal modes by means of a heterodyne process.

From Fig. 5, where the spectra for the 3 fiber lasers schemes are depicted, some conclusions can be drawn: firstly, the OSNR is around $60 \mathrm{~dB}$ in any of the 3 cases; and, secondly, the narrowest full width at half maximum is achieved for the purely ring fiber laser. Thus, this last result shows clear signs of that any presence of random and its associated Rayleigh scattering has a negative impact in the width of the laser as it was to be expected.
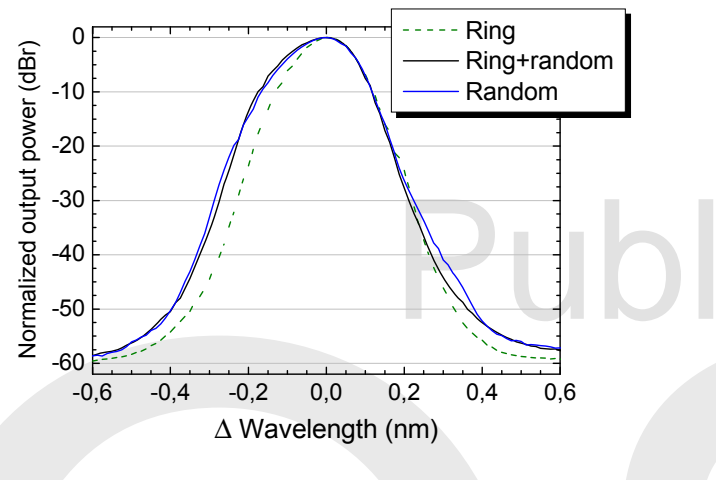

Fig. 5. Spectra for all 3 fiber lasers using normalized output power and normalized wavelength to show the differences among them in terms of OSNR and full width at half maximum.

Fig. 6 presents the longitudinal modes beat. When the Raman pump is $510 \mathrm{~mW}$, it can be said that the cavity behavior is purely a ring because the random laser has a higher lasing threshold and its influence is negligible in the modal evolution. However, when the Raman pump increases the spectrum is affected by the random mirror effect. On the one hand: the ring has a main role and modes are generated at the same frequencies; but on the other hand, the random process has certain impact on the behavior: the longitudinal modes are wider and wider and, also, the noise floor increases.

A completely different situation can be seen in Fig. 7, where the study of the longitudinal modes of the purely random fiber laser is shown. In this case, any longitudinal mode beat signal is observed in the picture in spite of the fact that the selected bandwidth is wide enough to address three beating modes. This modeless property is a unique characteristic of random fiber laser which is the main difference between ring fiber lasers and random fiber lasers $[27,28]$. It confirms the different nature of the fiber lasers under study.

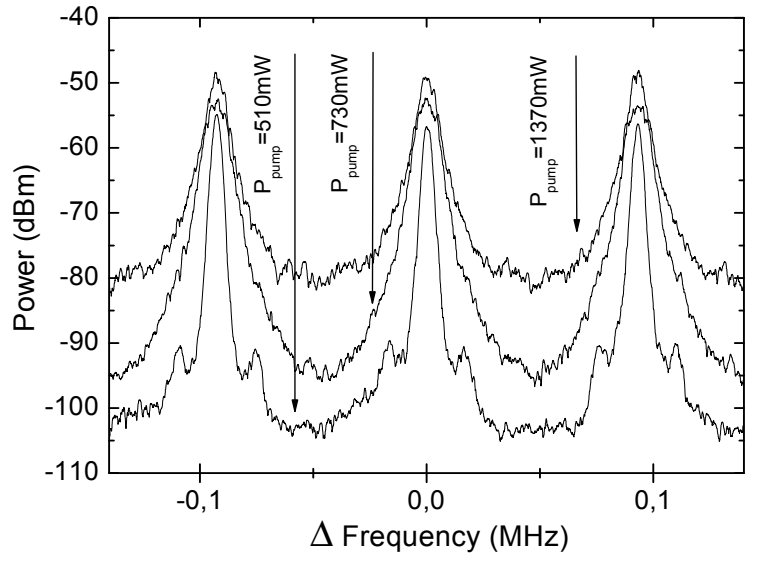

Fig. 6. Longitudinal modes generated in the hybrid ring-random fiber ring laser shown by the electrical spectrum analyzer.

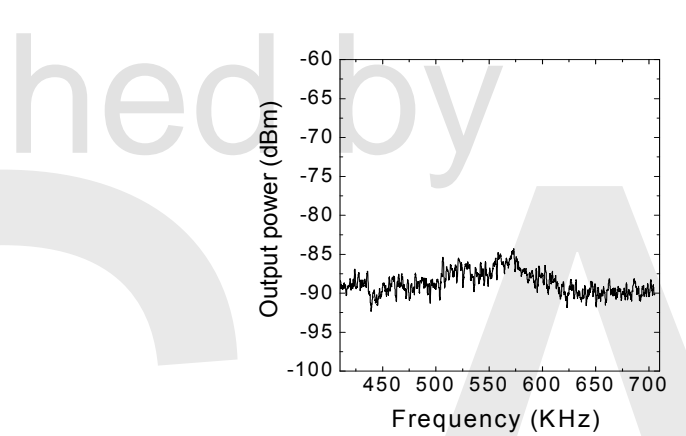

Fig. 7. Study of the beat modes in the case of the random fiber laser resulting in a modeless spectrum, characteristic of random fiber lasers.

For sake of completeness, it seems really important to demonstrate that the hybrid random-ring fiber laser is not just a conventional ring cavity fiber Raman laser with some impact of Rayleigh scattering but it is really composed of the contribution of two fiber lasers with different nature. Set-up shown in Fig. 8 is used to carry out this demonstration; it is the same scheme of the hybrid random-ring fiber laser presented in Fig. 1 (a) but taking into account both outputs of the $95 \%$ coupler.

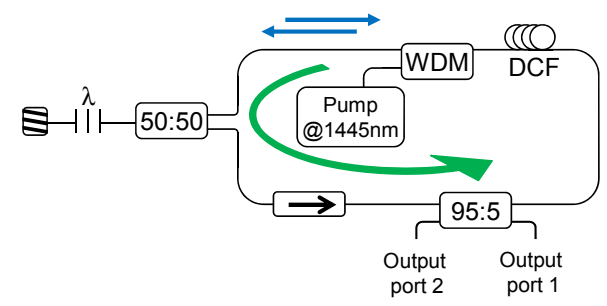

Fig. 8. Schematic set-up for the hybrid random-ring fiber laser in order to demonstrated that this laser is composed of the contribution of the ring fiber laser and the random fiber laser.

Fig. 9 (a) is the key of the demonstration; it shows the electrical spectra of both output ports simultaneously. On the one hand, output port 1 (depicted in green) shows the hybrid random-ring fiber laser, as there is ring component the resonances longitudinal modes appeared equidistant inversely proportional to the laser length. However, on the other hand, in output port 2 (depicted in blue) there are no longitudinal modes but the power 
level is higher than the receptor noise (depicted in black). So, the modeless spectra corroborates that there is a random fiber laser in the cavity along with the ring fiber laser. To finish, the optical spectrum of the output port 2 has been also studied. Fig. 9 (b) shows that there is also a laser emission in output port 2, which does not belong to the ring fiber laser because the ring fiber laser travels in the opposite direction; thus, there is a random fiber laser in the cavity. In conclusion, hybrid fiber laser is composed of the contribution of the ring fiber laser and the random fiber laser and it is not just a conventional ring cavity fiber Raman laser with some impact of Rayleigh scattering.

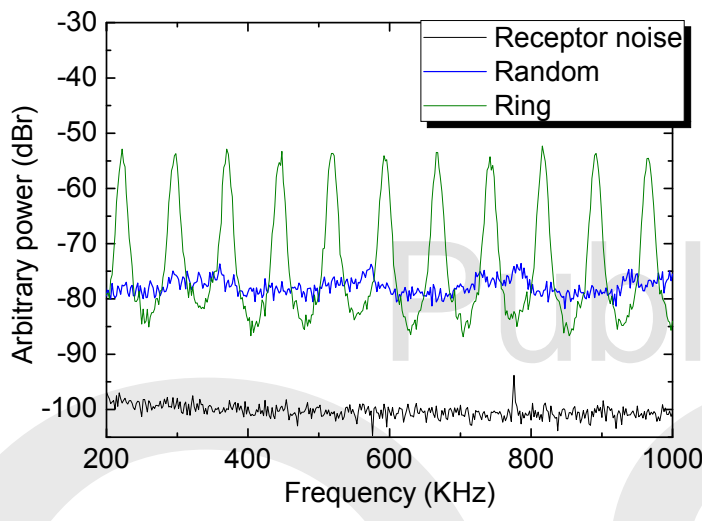

(a)

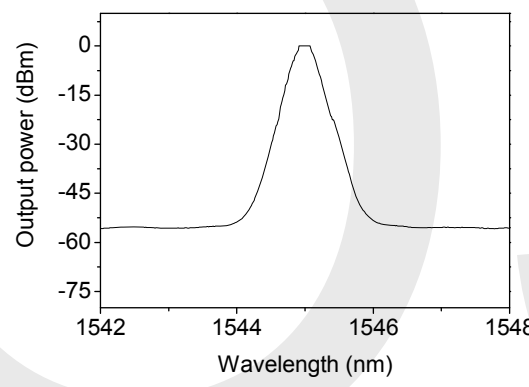

(b)

Fig. 9. Study of the (a) electrical and (b) optical spectra in order to demonstrate that the hybrid random-ring fiber laser is not just a conventional ring cavity fiber Raman laser with some impact of Rayleigh scattering but it is really composed of the contribution of the ring fiber laser and the random fiber laser.

Finally, the last part of the experimental section is devoted to the possible applications of the fiber lasers in the optical communication field. To accomplish this task, light was internally modulated by a LiNb03 Mach-Zehnder EOM (ElectroOptical Modulator) with an extinction ratio of $\sim 20 \mathrm{~dB}$ and the signal selected to modulate the structure was a pulsed shape one. In order to modulate internally the signal of both the ring and hybrid ring-random fiber laser, the electro-optical modulator has been directly included in the scheme, as shown in Fig. 10. Conversely, the random configuration was modified to modulate its signal because our electro-optical modulator is not a bidirectional component, so a 4 ports circulator was included in order to enable the back and forth path of the random signal.

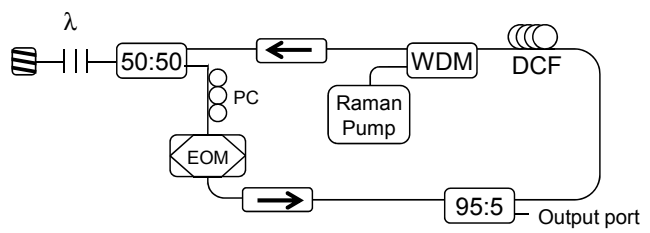

(a)

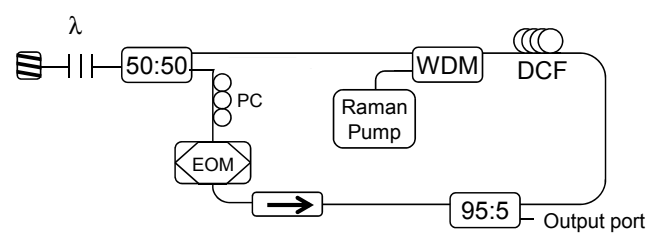

(b)

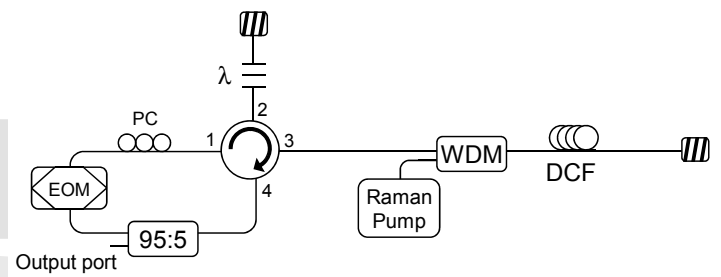

(c)

Fig. 10. Position of the electro-optical modulator in the three fiber lasers in order to modulate their signals: (a) hybrid ring-random fiber laser; (b) ring fiber laser and (c) random fiber laser. PC: polarization controller. EOM: electro-optical modulator.
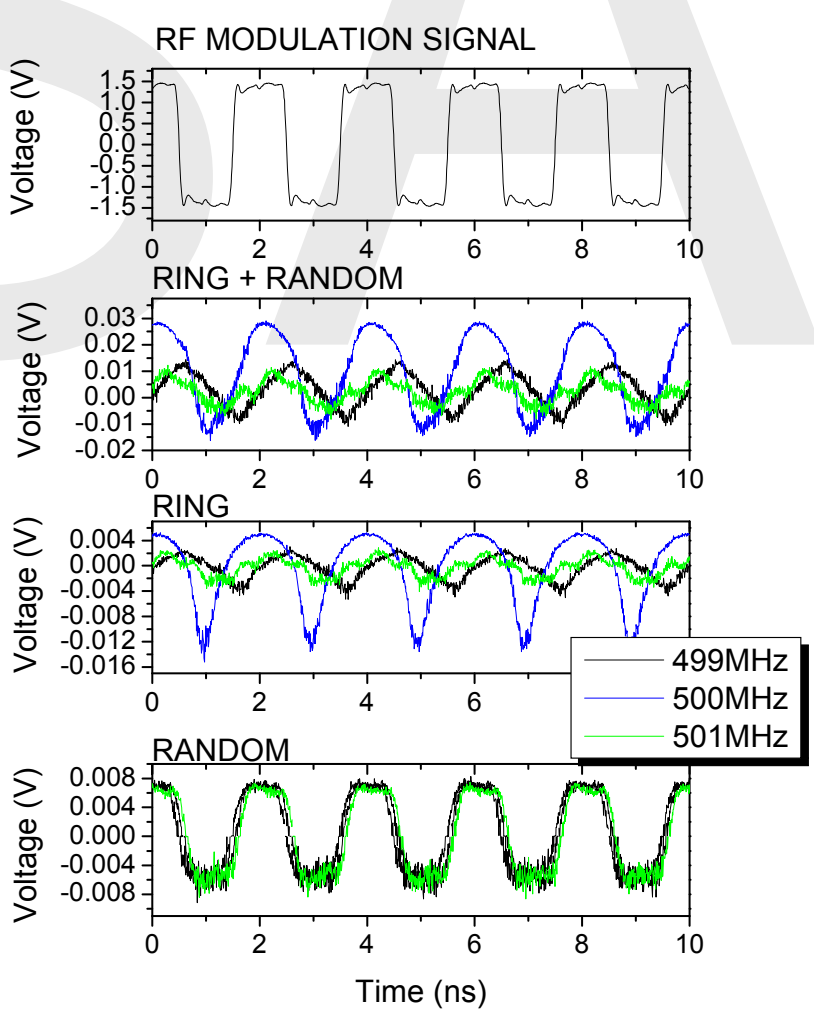

Fig. 11. Original modulation signal and time domain lasers behavior when $500 \mathrm{MHz}$ square signal internally modulated the signals of the different proposed fiber lasers. 
The frequency response characterization of a ring and a random cavity was demonstrated in [29], however in this paper we present a time-domain comparison to analyze the modulation distortion of the three configurations. Thus, in the particular case of modulate with a frequency of $500 \mathrm{MHz}$, in the random fiber laser the signal is not distorted as can be seen in Fig. 11, the same happens when the frequency is changed to $499 \mathrm{MHz}$ or 501 MHz. This fact means these kinds of lasers are able to adapt their cavities without distortion to each selected frequency of modulation. However, ring fiber lasers or hybrid ring-random fiber lasers with the ring laser having a dominant role have a conventional behavior: the length of the cavity is a decisive parameter because of the fact that it determines the possible repetition rate [29-32] if the laser is internally modulated. Taking into consideration this explanation, when one chooses a modulation frequency arbitrarily, the signal is completely distorted as can be seen in Fig. 11: the modulation is optimized when the frequency is $500 \mathrm{MHZ}$ where the distortion is evident, but, this effect is more significant when the frequency is changed only $1 \mathrm{MHz}$ up or down, in these cases the distortion is complete.

\section{Conclusions}

The behavior of three basic fiber lasers configurations has been experimentally evaluated in order to establish the main pros and cons of each scheme. The first fiber laser is based on a hybrid ring-random fiber laser; the second one is a purely ring fiber laser and the last one is a random fiber laser. Several aspects have been taken into account in the study: from the optical point of view, and the possible applications of the fiber lasers in the optical communication field.

Summarizing, firstly, the combination of both regimes of resonance, ring and random has a positive effect on the threshold power but a negative consequence in the output power and slope efficiency in comparison with the purely ring cavity. Secondly, the ring cavity offers the better stability, and the worst case belongs to the hybrid ring-random fiber laser. Thirdly, the different nature of the basic fiber lasers has been evaluated through the study of the longitudinal modes: in the ring cavity fiber laser and in the hybrid ring-random fiber laser, the cavity generates longitudinal modes equally spaced; however, the random fiber laser is characterized by a modeless behavior. Finally, it has been demonstrated that the random fiber lasers are able to adapt their cavities to any frequency of internal modulation, in contrast with ring fiber laser or hybrid ringrandom fiber lasers whose repetition rate is completely determined by the length of the cavity.

\section{Acknowledgements}

The authors would like to acknowledge the financial support from the Spanish Ministerio de Educación y Ciencia through project TEC2010-20224-C02-01

\section{References}

[1] C. Headly, Raman Fiber Lasers in Raman Amplifiers for Telecommunication 2, Sub-Systems and Systems (USA: M. N. Islam, 2003).
[2] C. Headly, Cascaded Raman Resonators in Raman Amplification in Fiber Optical Communication Systems (Oxford, UK: C. Headley and G. P. Agrawal, 2005).

[3] X. Dong, P. Shum, N. Q. Ngo and C. c. Chan, "Multiwavelength Raman fiber laser with a continuously-tunable spacing", Optics Express, 14(8), 3288-3293 (2006)

[4] J.D. Ania-Castañón, T. J. Ellingham, R. Ibbotson, X. Chen, L. Zhang, S. K. "Ultralong Raman fiber lasers as virtually lossless optical media," Physical Review Letters, 96(2), 023902-1-4 (2006)

[5] O. Frazão, C. Correia, M.T.M. Rocco Giraldi, M.B. Marques, H.M. Salgado, M.A.G. Martinez, J.C.W.A. Costa, A.P. Barbero and J.M. Baptista, "Stimulated Raman scattering and its applications in optical communications and optical sensors," The Open Optics Journal 3, 1-11 (2009).

[6] S. K. Turitsyn, J. D. Ania-Castañon, S. A. Babin, V. Karalekas, P. Harper, D. Churkin, S. I. Kablukov, A. e. El-taher, e. V. Podivilov and V. K. Mezentsev, "270-km ultralong Raman fiber laser," Phys. Rev. Lett., 103(13), 133901-1-4 (2009).

[7] C. Kim, R. M. Sova, J. U. Kang and J. B. Khurgin, "Novel multiwavelength cascaded-Raman source based on tunable fiber sagnac loop filter," in Proc. Conference on Optical Fiber Communication, Technical Digest Series, 242-243 (2002).

[8] Y. Han, J. H. Lee, S. H. Kim and S. B. Lee, "Tunable multi-wavelength Raman fibre laser based on fibre Bragg grating cavity with PMF LyotSagnac filter," Electron. Lett. 40(23), 1475-1476 (2004).

[9] M. Fernández-Vallejo, S. Diaz, R. A. Pérez-Herrera, R. Unzu, M. A. Quiniela, J. M. López-Higuera and M. López-Amo, "Comparison of the stability of ring resonator structures for multiwavelength fiber lasers using Raman or Er-Doped fiber amplification," IEEE J. Quant. Electron. 45(12), 1551-1557 (2009).

[10] A. E. El-Taher, P. Harper, S. A. Babin, D. V. Churkin, E. V. Podivilov, J. D. Ania-Castanon and S. K. Turitsyn, "Effect of Rayleigh-scattering distributed feedback on multiwavelength Raman fiber laser generation," Opt. Lett. 36(2), 130-132 (2011).

[11] M. Fernandez-Vallejo, D. Leandro, A. Loayssa and M. Lopez-Amo, "Fiber Bragg grating interrogation technique for remote sensing $(100 \mathrm{~km})$ using a hybrid Brillouin-Raman fiber laser," in Proc. of SPIE 7753 (2011).

[12].H. Cao, "Review on latest developments in random lasers with coherent feedback," J. Phys. Math. Gen. 38(49), 10497-10535 (2005).

[13] J. D. Ania-Castañón, T. J. Ellingham, R. Ibbotson, X. Chen, L. Zhang and S. K. Turitsyn, "Ultralong Raman fiber lasers as virtually lossless optical media," Phys. Rev. Lett. 96(2), 023902-1-4 (2006).

[14] D. S. Wiersma, "The physics and applications of random lasers," Nat. Phys. 4(5), 359-367 (2008).

[15] S. K. Turitsyn, J. D. Ania-Castañón, S. A. Babin, V. Karalekas, P. Harper, D. Churkin, S. I. Kablukov, A. E. El-Taher, E. V. Podivilov and V. K. Mezentsev, "270-km Ultralong Raman Fiber Laser," Phys. Rev. Lett. 103(13), 3901-1-4 (2009).

[16] S. K. Turitsyn, S. A. Babin, A. E. El-Taher, P. Harper, D. V. Churkin, S. I. Kablukov, J. D. Ania-Castañón, V. Karalekas and E. V. Podivilov, "Random distributed feedback fibre laser," Nature Photonics 4, 231235 (2010).

[17].A. A. Fotiadi, "Random lasers - an incoherent fibre laser," Nat. Photonics 4(4), 204-205 (2010).

[18] A. M. R. Pinto, O. Frazão, J. L. Santos and M. López-Amo, "Multiwavelength Raman fiber lasers using Hi-Bi photonic crystal fiber loop mirrors combined with random cavities," J. Lightwave Technol. 29(10), 1482-1488 (2011).

[19] H. Martins, M. B. Marques and O. Frazão, "300 km-ultralong Raman fiber lasers using a distributed mirror for sensing applications," Optics Express 19(19), 18149-18154 (2011).

[20] A. Pinto, M. Bravo, M. Fernandez-Vallejo, and Manuel Lopez-Amo, "Suspended-core fiber Sagnac combined dual-random mirror Raman fiber laser," Optics Express, 19(12), 11906-11915 (2011).

[21] I. D. Vatnik, D. V. Churkin and S. A. Babin, "Power optimization of random distributed feedback fiber lasers," Optics Express 20(27), 28033-28038 (2012).

[22] Y. J. Rao, Z. N. Wang, and X. H. Jia, "Output Characterization of Random Fiber Laser Formed by Dispersion Compensated Fiber," IEEE Photonics Technology Letters, 26(3), 246-248 (2014).

[23] Y. G. Han, C. S. Kim, J. U. Kang, U. C. Paek, and Y. Chung, "Multiwavelength Raman fiber ring laser based on tunable cascaded 
long-period fiber gratings," IEEE Photon. Technol. Lett. 15(3), 383385 (2003).

[24] Y. Han, T.V.A. Tran, S. Kim, S.B. Lee, "Multiwavelength Ramanfiber-laser-based long distance remote sensor for simultaneous measurement of strain and temperature," Opt. Lett. 30(11), 1282-1284 (2005).

[25] M Fernandez-Vallejo, S Diaz, R A Perez-Herrera, D Passaro, S Selleri, M A Quintela, J M Lopez Higuera and M Lopez-Amo, "Resilient longdistance sensor system using a multiwavelength Raman laser," Meas. Sci. Technol. 21(9), 094017 (5pp) (2010).

[26] M. Lopez-Amo, M. Fernandez-Vallejo, and D. Leandro, "Bidirectional Dual-Wavelength Raman Fiber Ring Laser," IEEE Photonics Technology Letters 23(7), 399-401 (2011).

[27] S. A. Babin, A. E. El-Taher, P. Harper, E. V. Podivilov and S. K. Turitsyn, "Tunable random fiber laser," Physical Review A - Atomic, Molecular and Optical Physics 84(20), 021805-1-4 (2011).

[28] Y. J. Rao, W. L. Zhang, J. M. Zhu, Z. X. Yang, Z. N. Wang and X. H. Jia, "Hybrid lasing in an ultra-long ring fiber laser," Optics Express, 20, 22563-22568 (2012).

[29] B. Vizoso, M. C. Vázquez, R. Civera, M. López-Amo, M. A. Muriel, "Amplified fiber-optic delay lines," Journal of Lightwave Technology, 12(2), 294-305 (1994).

[30] B. Dong, J. Hao, J. Hu and C. Liaw, "Short linear-cavity Q-switched fiber laser with a compact short carbon nanotube based saturable absorber," Optical Fiber Technology 17(2), 105-107 (2011).

[31] C. E. S. Castellani, E. J. R. Kelleher, Z. Luo, K. Wu, C. Ouyang, P. P. Shum, Z. Shen, S. V. Popov and J. R. Taylor, "Harmonic and single pulse operation of a Raman laser using graphene," Laser Physics Letters 9(3), 223-228 (2012).

[32] M. Bravo, M. Fernandez-Vallejo and M. Lopez-Amo, "Internal modulation of a random fiber laser," Opt. Lett. 38(9), 1542-1544 (2013). 